\title{
Understanding Personal Health Record and Facilitating its Market
}

\author{
Yujin Park ${ }^{1}$, Hyung-Jin Yoon ${ }^{1,2}$ \\ ${ }^{1}$ Department of Biomedical Engineering, Seoul National University College of Medicine, Seoul, Korea \\ ${ }^{2}$ Institute of Medical and Biological Engineering, Medical Research Center, Seoul National University, Seoul, Korea
}

The term "Personal Health Record" (PHR) has been used with a wide range of meanings for quite a long time [1]. PHR is a different concept from Electronic Medical Record (EMR; personal health-related electronic records managed by an authorized medical institution) or Electronic Health Record (EHR; personal health-related electronic records created and maintained by a number of authorized medical institutions that comply with national interoperability standards). PHR can be generally defined as "an electronic and lifelog record of health-related information that is provided and can be managed by an individual. The information can be stored and controlled with nationally recognized interoperability standards, even though it is drawn from multiple sources." [2-5].

With a clear concept of PHR, there is a growing interest in the availability and benefits of PHRs. PHRs will enhance not only the quality of the healthcare environment, but also the

Submitted: February 10, 2020

Revised: June 15, 2020

Accepted: July 1, 2020

\section{Corresponding Author}

Hyung-Jin Yoon

Department of Biomedical Engineering, Seoul National University College of Medicine, 103 Daehak-ro, Jongno-gu, Seoul 03080, Korea. Tel: +82-2-2072-7516, E-mail: hjyoon@snu.ac.kr (https://orcid. org/0000-0003-4432-4894)

This is an Open Access article distributed under the terms of the Creative Commons Attribution Non-Commercial License (http://creativecommons.org/licenses/by$\mathrm{nc} / 4.0 /$ ) which permits unrestricted non-commercial use, distribution, and reproduction in any medium, provided the original work is properly cited.

(C) 2020 The Korean Society of Medical Informatics patients' knowledge of personal health-related information. This paper will explore further attention points for facilitating the PHR market with its characteristics and strengths.

\section{Characteristics of the PHR Market}

\section{What are the Characteristics of a PHR?}

The characteristics of a PHR include the following. It is a personal lifetime record that is integrated and comprehensive. It is owned, managed and shared by an individual, and it can be accessed anytime, anywhere. It is provided by individuals or by other medical institutions, and it is not a legal document [1]. Considering these characteristics, when a service utilizing a PHR is implemented, the patient may be at the center of the medical environment.

\section{Why is PHR Service Important and Who Benefits?}

PHR services enable patients to actively participate in the preventative healthcare environment based on daily health information [6]. Moreover, they can meet the demands of patients who have moved from post-treatment to proactivetreatment [1]. However, because the benefits of using PHR services are not yet clear, potential PHR users have low demand to participate in the PHR business model [7].

Both patients and clinicians can expect benefits from using PHRs [8]. First, patients can manage and access their health data; this allows them to participate in medical decisionmaking beyond the current healthcare environment, which relies unilaterally on health professionals. In particular, patients with chronic diseases can expect better quality of care with convenient access to medical services and health 
professionals. Moreover, the ability to track a patient's lifestyle or health behaviors in addition to their hospitalization or medication history makes it easier for clinicians to offer personalized care while reducing consultation time $[1,6]$.

\section{Requirements for Facilitating the PHR Market}

\section{Data Standards and Related Regulations for Vitalizing the PHR Market}

PHR data standards should take into account both adherence to international standards and actual domestic medical information conditions so that domestic PHR service providers can cultivate their global market competitiveness. If Korea can propose government-led PHR standards and guidelines similar to the Precision Medicine Initiative and Blue Button Initiative in the United States, it can be expected that domestic companies' medical information technology will increase to an international level.

Certain levels of incentives are required for healthcare providers or patients to actively participate in PHR services. The Office of the National Coordinator for Health Information Technology (ONC) established standards on interoperability and security for medical information exchange and introduced an incentive-certified system [9]. The incentive system is administered by the Centers for Medicare and Medicaid Services (CMS) to give healthcare providers defined incentives when they meet the phase-by-step performance management items presented by the ONC. Clear definitions of health-related legislative readjustments are also needed, such as collection and utilization upon consent, personal health records managed by external companies, and telemedicine activities [10].

\section{Data Integrity}

Because PHR data is collected by various devices in a variety of formats, data integrity is important to keep information complete and accurate in the process of transmission [11]. As the information systems handling personal health information change on each unit, it is essential to ensure the integrity of the data and systems by defining sequential procedures to prevent new risks or vulnerabilities and to perform format changes $[7,12]$.

\section{User Health Literacy Technology and Development Support for PHR}

The biggest obstacle to PHR market activation is that it is difficult for users to realize the benefits of managing their health records, and various applications are required for them to be able to manage their own health records. In addition, users need to understand their PHR and utilize it to manage their healthcare, so called health literacy, which can be achieved through the development of smartphone applications combined with data visualization technologies based on artificial intelligence or virtual reality technologies $[9,13]$. The government should provide functional and cognitive support with technology development to improve health literacy for both patients and caregivers while enhancing the spread of PHR-related services and technology.

\section{Conflict of Interest}

No potential conflict of interest relevant to this article was reported.

\section{Acknowledgments}

This work was supported by the Technology Innovation Program (20003057, Development of PHR data standards and guidelines to improve market competitiveness) funded By the Ministry of Trade, Industry \& Energy (MOTIE, Korea).

\section{ORCID}

Yujin Park (http://orcid.org/0000-0002-7936-9307)

Hyung-Jin Yoon (http://orcid.org/0000-0003-4432-4894)

\section{References}

1. Heo Y, Yang JS, Park KH, Cha SJ, Choi DJ, Hwang KH. Personal health record (PHR) service technology and industry trends. KIET PD Issue Rep 2013;13(11):70-96.

2. Burrington-Brown J, Fishel J, Fox L, Friedman B, Giannangelo K, Jacobs E, et al. Defining the personal health record. AHIMA releases definition, attributes of consumer health record. J AHIMA 2005;76(6):24-5.

3. Collman J, Demster B. Managing information privacy and security in healthcare [Internet]. Chicago (IL): Healthcare Information and Management Systems Society; 2013 [cited at 2020 Jul 11]. Available from: https:// www.himss.org/sites/hde/files/d7/HIMSSorg/Content/ files/CPRIToolkit/version6/v7/D01a_History_PSToolkit.pdf.

4. The Office of the National Coordinator for Health Information Technology. What are the differences between electronic medical records, electronic health 
records, and personal health records? [Internet]. Washington (DC): The Office of the National Coordinator for Health Information Technology; 2019 [cited at 2020 Jul 11]. Available from: https://www.healthit.gov/faq/whatare-differences-between-electronic-medical-recordselectronic-health-records-and-personal.

5. The National Alliance for Health Information Technology Report to the Office of the National Coordinator for Health Information Technology on defining key health information technology terms. Washington (DC): Department of Health and Human Services; 2008.

6. Prashad R. The role of personal health record systems in chronic disease management. Stud Health Technol Inform 2017;234:275-9.

7. Detmer D, Bloomrosen M, Raymond B, Tang P. Integrated personal health records: transformative tools for consumer-centric care. BMC Med Inform Decis Mak 2008;8:45.

8. Tang PC, Ash JS, Bates DW, Overhage JM, Sands DZ. Personal health records: definitions, benefits, and strat- egies for overcoming barriers to adoption. J Am Med Inform Assoc 2006;13(2):121-6.

9. Ford EW, Hesse BW, Huerta TR. Personal health record use in the United States: forecasting future adoption levels. J Med Internet Res 2016;18(3):e73.

10. Shin SY, Chung CK. A future of medical information system: establishment of hospital-oriented personal health record. J Korean Med Assoc 2009;52(11):111521.

11. Chen TS, Liu CH, Chen TL, Chen CS, Bau JG, Lin TC. Secure Dynamic access control scheme of PHR in cloud computing. J Med Syst 2012;36(6):4005-20.

12. Korea Health Industry Development Institute. Information security guide for medical institutions. Cheongju, Korea: Korea Health Industry Development Institute; 2016.

13. Stead WW, Lin HS. Computational technology for effective health care: immediate steps and strategic directions. Washington (DC): National Academies Press; 2009. 